\title{
Impacto De La Realidad Aumentada En La Educación Del Siglo XXI
}

\author{
Franklin Montecé-Mosquera, Profesor
}

Universidad Regional Autónoma de los Andes (UNIANDES), Ecuador

Alexis Verdesoto-Arguello, Profesor

Instituto Técnico Superior Eugenio Espejo, Ecuador

Carlos Montecé-Mosquera, Ingeniero en Administración de

Empresas

Universidad ECOTEG , Ecuador

Cesar Caicedo-Camposano, Abogado

Universidad Técnica de Babahoyo, Ecuador

doi: 10.19044/esj.2017.v13n25p129 URL:http://dx.doi.org/10.19044/esj.2017.v13n25p129

\begin{abstract}
The vertiginous advances in terms of information anda communication technologies mean allow innovative ways to stregthen aspects related to knowledge. With the passing of the last years the Augmented Reality RA has positioned itself as a technology that has approached different environments of society, environments related to advertising, marketing, video games and in education has generated a high impact. Augmented reality is technology that contributes resources to the world, it seeks to determine if the incorporation of technologies contribute an enrichment to the teaching-learning process.

Considering the analysis of works related to the theme, the reality may be $\mathrm{d}$ efined as that additional information obtained by observation of an environment with the objective to innovate the teaching process by providing a tool that presents the user: interaction, entertainment and motivation learning.
\end{abstract}

Keywords: Android, Education, Innovation, Augmented Reality, Technology Mobile, TicsIntroducción

\section{Resumen}

Los vertiginosos avances en lo que a las tecnologías de la información y la comunicación se refiere, permiten formas innovadoras para afianzar aspectos relacionados con el conocimiento. Con el transcurrir de los últimos 
años la Realidad Aumentada (Augmented Reality) RA se ha posicionado como una tecnología que se ha acercado a diferentes entornos de la sociedad, entornos relacionados con la publicidad, el mercadeo, videojuegos y en la educación ha generado un alto impacto. La realidad aumentada es una tecnología que aporta unos recursos al mundo, se busca determinar si la incorporación de tecnologías aportan un enriquecimiento al proceso enseñanza - aprendizaje. Considerando el análisis de trabajos relacionados con el tema, la realidad aumentada podría definirse como aquella información adicional que se obtiene de la observación de un entorno con el objetivo de innovar el proceso de enseñanza brindando una herramienta que presente al usuario: interacción, entretenimiento y motive su aprendizaje.

Palabras-Claves: Android, Educación, Innovación, Realidad Aumentada, Tecnología Móvil, Tics

Las aplicaciones con Realidad Aumentada (RA) tienen su auge aproximadamente en el año 2002, con la evolución de las tecnologías de la información y las comunicaciones (Tics), que se implementaron en dispositivos móviles y se desarrollaron aplicaciones colaborativas con RA. (Posada, 2014).

De la revisión bibliográfica realizada además de la información obtenida de la consulta a expertos se logra entender que la realidad aumentada está ganando terreno constantemente y lo hace mostrando sus grandes beneficios en diferentes entornos, así como en el ámbito educativo, ya existen portales que proponen al docente el uso de herramientas tecnológicas en el aula. Su implementación en los diferentes niveles y disciplinas ha sido posible gracias a la diversidad de aplicaciones y software de RA que se están diseñando, donde el contenido de aprendizaje se muestra en modalidad virtual para complementar el contexto real y enriquecerlo. La información adicional identificada como realidad aumentada puede traducirse en diferentes formatos por ejemplo: Puede ser una imagen, un carrusel de imágenes, un archivo de audio, un vídeo o un enlace. Es conocido que los docentes usan diversas técnicas en el proceso de enseñanza, con el enfoque colaborativo, donde los integrantes comparten información, inquietudes, conocimientos e intercambios de ideas por lo tanto la aplicación de tecnología es una puerta abierta a la espera del usuario y al creciente desarrollo de ellas.

"La tecnología se diseña como un componente de la identidad humana, una de sus expansiones constitutivas. [...] A diferencia de los animales que, en muchos casos, muestran sus habilidades técnicas inscritas en su equipamiento biológico y adaptables al entorno dentro de un abanico de posibilidades predefinidas, el hombre tiene en su predisposición biológica 
la posibilidad de crear y usar tecnología" (Maragliano, 2008, pp. 7).

Los autores (Pedro \& Martinez, 2012) plantean que la RA es una alternativa metodológica en la educación primaria y parten de la idea de que toda herramienta multimedia que sirva para presentar material académico con fines educativos, complementan los métodos de enseñanza tradicionales.

El trabajo de (García \& Cabero, 2016) presenta a la RA como una de las tendencias de uso que se impone y que tienen una presencia significativa en los aspectos relacionados con la formación. El objetivo del presente trabajo de investigación es pretender que el lector encuentre motivación para direccionar todo avance tecnológico que se obtenga con la realidad aumentada a la formación académica, determinando que sirva de apoyo para el proceso educativo.

En 2002 se publicó el reporte "2020 Visions, Transforming Education and Training Through Advanced Technologies", en español, "Visiones, transformando la educación y el entrenamiento a través de tecnologías avanzadas" que comprende una serie de artículos que plantean escenarios factibles para el año 2020 referidos a los usos de las TIC en las instituciones educativas. En ellos se plantea la transformación de la vida cotidiana de los estudiantes en las escuelas, la innovación en los métodos de enseñanza, los materiales educativos y la evaluación, así como el cambio radical de lo que hoy concebimos como espacio físico de aula y, por supuesto, la emergencia de nuevas demandas a la capacitación y funciones del profesorado.

En el último año (Hanke, 2016) se popularizó un juego de realidad aumentada para atrapar pokemones virtuales llamada Pokemon Go, en contextos reales, el que se ha vuelto ya un fenómeno mundial con más de 15 millones de usuarios. El videojuego permite cumplir -más bien recrear- un sueño que en nuestra infancia creíamos irrealizable: atrapar pokémons tal como lo hacían los personajes de la serie. El doctor en psicología Juan Moisés de la Serna, autor de Ciberpsicología: relación entre mente e Internet, habla de una "huella" en la memoria del consumidor que se reactiva cuando franquicias tan influyentes lanzan un producto nuevo (Serna, 2016).

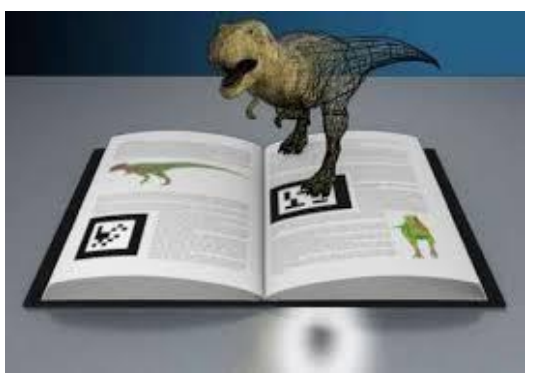

Figura1

Fuente: Serna, 2016 
El autor (Barriga, 2007) señala que "si valoramos la forma en que los desarrollos tecnológicos se han ido introduciendo en el campo de la educación (Figura 1), vemos que ha impactado de manera significativa la educación, en el sentido de que se está propiciando un cambio profundo en los paradigmas educativos" (p. 3).

Se considera que el aporte de este proyecto es de relevancia, debido a que prepara al lector con fundamentos teóricos y prácticos actualizados, que brindan una base para generar nuevas propuestas o ideas innovadoras en el área de la educación.

\section{Métodos}

Esta investigación se fundamenta en la modalidad cualitativa porque se basa en el análisis teórico de información relacionada con la Realidad Aumentada, la Investigación fue documental ya que el problema planteado necesitó de sustento teórico es así que la investigación se fundamentó en artículos científicos publicados en revistas tecnológicas indexadas desde el 2014 hasta el presente año, así como de libros de relevancia por su contexto, autor y actualidad, detallados en las referencias bibliográficas.

Se aplicó el método analítico en vista que fue necesario comprender el modelo de enseñanza de los docentes y observar su desempeño en el ámbito educativo. Fue necesario un estudio detallado del objeto de investigación para su posterior descomposición: funcionamiento, uso, aplicaciones, enfoque y alcance del objetivo general del proyecto. Se estudiaron las posibles aplicaciones en el alumnado y en las formas de uso para definir el funcionamiento más adecuado y su contribución. Basado en la posición teórica de (Barriga, 2007) puede afirmarse que cuando se valora el aporte que ha dado el avance tecnológico a la educación se podrán ver las posibilidades de cambio (Figura 2) que pueden ofrecerse mediante el trabajo de investigación e implementación de aportes prácticos mejorados para el ambiente educativo.

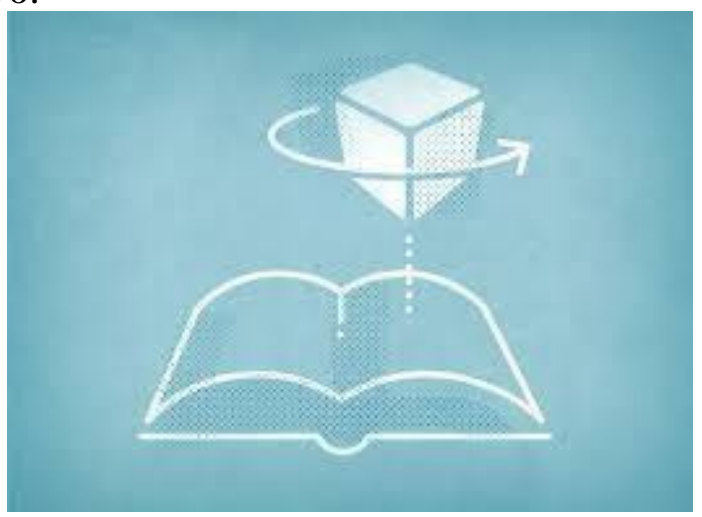

Figura 2

Fuente: Barriga, 2007 
Existe una propuesta que el proceso de programación se determinó utilizar la metodología de desarrollo de software AUP - Proceso Unificado Ágil, la cual es heredera de otros paradigmas como la programación extrema (XP); se desarrolla en fases y demanda un conjunto de iteraciones, se van emitiendo entregables y prototipos de software con miras a la culminación del producto.

Para la programación de la aplicación se investigó cuáles son las mejores herramientas para el desarrollo de realidad aumentada, teniendo como opciones de mayor relevancia: ARToolkit, ARPA, Layar, Unity 3D, Vuforia. En vista de que Unity 3D es un motor de desarrollo para videojuegos profesional, gratuito, compatible con $\mathrm{W}$ indows 8 y que funciona perfectamente con la librería de RA Vuforia, se determinó utilizar estas dos últimas, de acuerdo a la experiencia del autor.

Luego de la valoración respectiva se determina que este enfoque trae como beneficios la atenuación de riesgos desde ciclos tempranos del proceso, alineando las necesidades de los usuarios a las funcionalidades del producto y a su vez, promueve una correcta administración del cambio y la configuración.

A continuación se presenta la estructura del proyecto propuesto por estudiantes de la carrera de sistemas según metodología de proceso unificado ágil:

Tabla 1. Estructura de iteración

\begin{tabular}{|c|c|}
\hline I & Determinación de los lenguajes de programación y herramientas a utilizar \\
\hline II & Diseño y elaboración de marcadores en nivel de realidad aumentada I \\
\hline III & Diseño de la base de datos online en plataforma Vuforia \\
\hline IV & Diseño y preparación del contenido 3D \\
\hline V & Programación y pruebas de las funcionalidades de la aplicación \\
\hline VI & Diseño y elaboración de marcadores en nivel de realidad aumentada II \\
\hline VII & Programación y pruebas de las funcionalidades de la aplicación \\
\hline VIII & Diseño y elaboración de libro de realidad aumentada \\
\hline IX & Enriqueciendo información en tiempo real digitalizada \\
\hline $\mathbf{X}$ & Programación y pruebas de las funcionalidades de la aplicación \\
\hline XI & Adaptabilidad para Smartphone con sistema operativo android a partir de la \\
\hline XII & Programación y pruebas finales de las funcionalidades de la aplicación \\
\hline
\end{tabular}

Fuente: Los autores

Es importante recalcar que en el trabajo citado se intenta profundizar el proceso de enseñanza-aprendizaje, estudiar las características que influyen en el alumnado al utilizar contenidos de RA y las ventajas de mantener la atención del alumno y los mecanismos que se activan mediante su utilización. 


\section{Análisis e interpretación}

Las autoridades de planteles educativos están de acuerdo que el uso de tecnologías en el aula de clases es conveniente, consideran que todo aporte tecnológico que logre un cambio positivo en el ámbito educativo es bienvenido.

Algunas autoridades han escuchado acerca de la realidad virtual, pero no sobre la aumentada. Aunque existe desconocimiento de esta tecnología no presenta inconvenientes su uso y aplicación, debido a que es simple e intuitivo, concuerdan en que el continuo cambio y actualización del modo de enseñanza es importante debido al cambiante y creciente avance tecnológico. Una herramienta como la realidad aumentada es muy atrayente para los estudiantes, especialmente de esta edad donde lo novedoso gusta y, a su vez, logra innovar el proceso de enseñanza, opinaron las autoridades de la Unidad Educativa Monterrey de Babahoyo.

\section{Resultados}

En la implementación del proyecto se evidenció gran agrado e interés en el alumnado, al conocer el cambio en el paradigma de aprendizaje, haciendo uso de un teléfono móvil que permitió tener una mejor experiencia educativa al interactuar con objetos de tercera dimensión multimedia que se despliegan en la pantalla al enfocar el libro de RA, mostrando características relevantes del objeto según la asignatura o temática. Como se definió en la introducción, existen niveles de realidad aumentada, por lo tanto las iteraciones de desarrollo iniciaron desde el nivel 1 por medio de marcadores a blanco y negro que servirán de impulsador al ser reconocido por la cámara del celular del cual hace uso la app. No cualquier marcador puede servir, se necesita hacer pruebas y analizarlas mediante la plataforma online de Vuforia quien define si es aceptable o no para ser añadida a la base de datos. El diseño fue realizado en photoshop cs6, hasta lograr que sean trackables (rastreables) por vuforia.

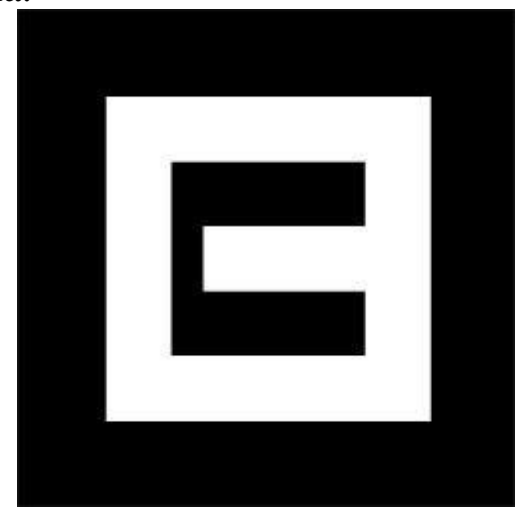

Figura 3

Fuente: Los autores 
Como siguiente avance de nivel 1, se estimó reemplazar los marcadores a blanco y negro por Códigos QR como marcadores para presentar la información digital, dado que presentan una gran aceptación por la plataforma vuforia; para agilizar el proceso se optó por utilizar un generador de códigos qr vía online en el sitio http://www.codigosqr.com/generador-de-codigos-qr/, combinando códigos qr y marcadores a blanco y negro. Así como resultado se obtuvo:

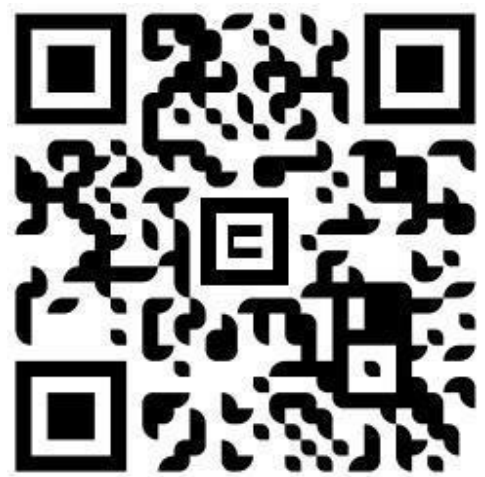

Figura 4: Segundo marcador combinando código QR y marcador inicial Fuente: Los autores

Mejorando el marcador para la base de datos trackable se combinó el logo de la aplicación con el diseño de código qr a colores, mejorando la calificación que otorga vuforia y la presentación de los marcadores. Una vez superado con éxito el nivel 1 con aproximadamente 35 compilaciones, se procedió a desarrollar en el nivel 2, realidad aumentada sin marcadores; es decir, se reemplazaron los marcadores por imágenes y formar así un libro de realidad aumentada que presente al alumno información detallada acerca de la materia a estudiar y a la vez pueda interactuar con objetos virtuales relacionados reflejados sobre el libro mediante la cámara del teléfono móvil.

\section{Conclusion}

La aplicación de la tecnología de la realidad aumentada al proceso de enseñanza- aprendizaje presenta ventajas respecto a los métodos tradicionales de enseñanza. El realismo, interactividad, motivación e interés en aprender son los factores más importantes a destacar, evidenciado en los alumnos a partir del uso de esta herramienta.

Se aporta una solución tecnológica móvil de bajo costo, con el fin de innovar el proceso de enseñanza donde los estudiantes podrán acceder a contenidos virtuales en tercera dimensión sobre los temas que están aprendiendo, generando un ambiente de trabajo diferente que motive a los alumnos a aprender. Se ofrece varias opciones de presentar los contenidos como: folletos con marcadores $\mathrm{QR}$, Cubos de códigos $\mathrm{QR}$ y libros 
aumentados.

Se considera que la unión de una o varias herramientas a la realidad aumentada podrían generar mejor obtención de resultados, independientemente del área de aplicación a la que sea sometida esta tecnología.

\section{References:}

1. Barrere, R., Martínez, E. C., Lucio, I. F., Gordo, A., Jacovkis, P., \& Polino, C. (2015). Ciencia, tecnología e innovación para el desarrollo y la cohesión social - Programa iberoamericano en la década de los bicentenarios. Madrid, España: OE.

2. Barriga, F. D. (2007). Educación y nuevas tecnologías de la información: ¿hacia un nuevo paradigma educativo innovador? (Universidad Nacional Autónoma de México ed.). México.

3. Cabero, J., \& Barroso, J. (2016). Ecosistema de aprendizaje con realidad aumentada: posibilidades educativas. Revista Tecnología Ciencia y Educación de Carácter Científico Multidisciplinar(Num. 5).

4. Cabero, J., \& Barroso, J. (2016). Posibilidades educativas de la Realidad Aumentada. Revista de Nuevos Enfoques en Investigación Educativa, $\quad$ Vol. $\quad 5 \quad$ (Num. doi:http://dx.doi.org/10.7821/naer.2016.1.140

5. Cadillo, J. (2011). Uso de la Realidad Aumentada en la Compresión de la Proceso Histórico: Libro Conociendo el Museo Arqueológico de Ancash.

6. Cubillo, J., Martín, S., Castro, M., \& Colmenar, A. (2014). Recursos digitales autónomos mediante realidad aumentada. (L. G. Aretio, Ed.) Revista Iberoamericana de la Educación Digital - RIED, Vol. 17(Num. 2).

7. Duart, J., \& Abaitua, C. (2014). Enseñar y aprender con las TIC. Revista de Estudios sobre educación, Vol. 20, 9-19.

8. Fernández, M. (2014). Las nuevas tecnologías en la educación y Análisis de modelos de aplicación. Universidad Autónoma de Madrid: Departamento de Didáctica y Teoría de la Educación.

9. García, S., \& Cabero, J. (2016). Realidad aumentada: Tecnología para la formación.

10. Revista de medio y comunicación Pixel-Bit (Num. 49).

11. Gómez, D. (2017). La innovación educativa basada en la tecnologia. Revista Científica Multi-ensayos, ciencia, tecnología y salud, Vol. 2 (Num. 4), 102-106.

12. Hanke, J. (2016). Fundador y Director de Pokemon Go, Google Earth, Niantic. 
13. Heilig, M. (1957). The Father of Virtual Reality. Los Angeles, California.

14. Hernández, R., Fernandez, C., \& Baptista, P. (2006). Metodologia de Investigación

15. (Cuarta Edición ed.). México D.F.: Mc Graw Hill.

16. Inciarte, M. (2016). Tecnologías de la Información y la Comunicación. Un eje transversal para el logro de aprendizajes significativos. Revista Iberoamericana sobre Calidad, Eficacia $y$ Cambio en Educación - REICE, Vol. 2(Num. 1). doi:10.15366/reice

17. Kato, H. (2010). ARToolKit biblioteca GNU GPL - Creador y desarrollador. Washington - Estados Unidos : Universidad de Washington.

18. Kaufmann, H. (2003). Colaborativo: Realidad aumentada en la educación. Proc. Imagina 2003.

19. Marimón, D. (2010). El futuro de la realidad aumentada móvil. Cuadernos de comunicación e Innovación.

20. Marín, V. (2016). Posibilidades de uso de la Realidad Aumentada en la educación inclusiva. Estudio de caso. Revista Ensayos - Facultad de Educación de Albacete, 31(2).

21. Pedro, D., Kato, M., \& Reinoso. (2014).

22. Pedro, J. D., \& Martinez, C. (Mayo de 2012). Realidad Aumentada: Una Alternativa Metodológica en la Educación Primaria Nicaragüense. Revista Iberoamericana de Tecnologías del Aprendizaje, Vol. 7 (Num. 2).

23. Posada, F. (2014). canaltic. Obtenido de Uso educativo de las TIC: http://canaltic.com/blog/?p=1859

24. Prendes, C. (2015). Realidad aumentada y educación: análisis de experiencias prácticas. Revista de Medios y Educación PixelBit(Num. 46). doi:http://dx.doi.org/10.12795/pixelbit.2015.i46.12

25. Rice. (2009). Augmented vision and the decade of ubiquity.

26. Rimari, W. (2015). La Innovación Educativa como un instrumento de desarrollo.

27. Ruiz, R. (2006). Historia y evolución del pensamiento científico. México. Serna, J. d. (2016). Relación entre mente e Internet.

28. Severin, E. (2013). Enfoques Estratégicos sobre Tics en Educación en América Latina y el Caribe. Santiago de Chile: Organización de las Naciones Unidas para la Educación la Ciencia y la Cultura.

29. Wilches, D. (2011). Visualizaciòn de Informaiciòn Urbana Geo referenciada por medio de Realidad Aumentada. 